\title{
Grüneisen parameters for the Lieb-Liniger and Yang-Gaudin models
}

\author{
Li Peng $\odot,{ }^{1,2}$ Yicong Yu $\odot,{ }^{1, *}$ and Xi-Wen Guan $\oplus^{1,3,4, \dagger}$ \\ ${ }^{1}$ State Key Laboratory of Magnetic Resonance and Atomic and Molecular Physics, Wuhan Institute of Physics and Mathematics, APM, \\ Chinese Academy of Sciences, Wuhan 430071, China \\ ${ }^{2}$ University of Chinese Academy of Sciences, Beijing 100049, China \\ ${ }^{3}$ Center for Cold Atom Physics, Chinese Academy of Sciences, APM, Wuhan 430071, China \\ ${ }^{4}$ Department of Theoretical Physics, Research School of Physics and Engineering, Australian National University, \\ Canberra ACT 0200, Australia
}

(Received 19 September 2019; revised manuscript received 15 November 2019; published 27 December 2019)

\begin{abstract}
Using the Bethe ansatz solution, we analytically study expansionary, magnetic, and interacting Grüneisen parameters (GPs) for one-dimensional (1D) Lieb-Liniger and Yang-Gaudin models. These different GPs elegantly quantify the dependence of characteristic energy scales of these quantum gases on the volume, the magnetic field, and the interaction strength, revealing the caloric effects resulting from the variations of these potentials. The obtained GPs further confirm an identity which is incurred by the symmetry of the thermal potential. We also present the universal scaling behavior of these GPs in the vicinities of the quantum critical points driven by different potentials. The divergence of the GPs not only provides an experimental identification of non-Fermi-liquid nature at quantum criticality but also elegantly determines low-temperature phases of the quantum gases. Moreover, the pairing and depairing features in the 1D attractive Fermi gases can be captured by the magnetic and interacting GPs, facilitating experimental observation of quantum phase transitions. Our results open the way to further study the interaction- and magnetic-field-driven quantum refrigeration and quantum heat engine in quantum gases of ultracold atoms.
\end{abstract}

DOI: 10.1103/PhysRevB.100.245435

\section{INTRODUCTION}

The Grüneisen parameter (GP), originally introduced to characterize the frequency change due to the variation of volume in a crystal lattice [1,2], plays an important role in the study of pressure and volume effects in solid-state materials. Usually, it was determined from the ratio of thermal expansion to specific heat, quantifying the pressure dependence of characteristic energy scales of solid materials. Nowadays, it is extensively studied in geophysics [3,4], plasma physics [5-7], chemistry physics $[8,9]$, and various fields of physics. The GP has recently been investigated in heavy-fermion systems [10-12].

The divergence of the GP in these systems shows generic signatures of quantum criticality $[13,14]$, revealing the adiabatic magnetocaloric effect of the heavy-fermion metals.

Dimensionless constants such as the Wilson ratio [15], Wiedemann-Franz law [16], and the Kadowaki-Wood ratio $[17,18]$ are very useful in the study of quantum liquids and electronic transport properties. The nature of these ratios essentially reflects the ratio between two types of fluctuations. It is well known that the susceptibility (compressibility) Wilson ratio proposed in $[15,19,20]$ presents the ratio between the polarization (or particle number) fluctuation and the energy fluctuation. Although there have been extensive studies on the GP in various fields of physics, little work is carried out on

\footnotetext{
*ycyu@wipm.ac.cn

†xwe105@wipm.ac.cn
}

the GP for the quantum gases. This is mainly because both thermal expansion and specific heat in quantum gases [21] are notoriously difficult to be measured in experimental study. A recent study [22] shows that the magnetocaloric effect (or interaction-driven caloric effect) can help measure the GPs in the controllable systems of ultracold atoms.

The GP parameter $[1,2]$ reveals the spectrum change (anharmonicity of the frequency) to the variation of the volume of a crystal lattice. However, in literature, the formulation of the GP seems to be miscellaneous for different physical phenomena. By the definition of the GP, we first present an explicit form of the GP in grand canonical ensemble [22]

$$
\Gamma=\frac{\left.V \frac{d p}{d T}\right|_{V, N}}{\left.\frac{d E}{d T}\right|_{V, N}}=\frac{1}{T} \frac{\frac{\partial^{2} p}{\partial \mu^{2}} \frac{\partial p}{\partial T}-\frac{\partial^{2} p}{\partial \mu \partial T} \frac{\partial p}{\partial \mu}}{\frac{\partial^{2} p}{\partial \mu^{2}} \frac{\partial^{2} p}{\partial T^{2}}-\left(\frac{\partial^{2} p}{\partial \mu \partial T}\right)^{2}},
$$

where the Maxwell relations and general thermal relation were used. This expression together with relation (5) presents a quantitative description of the caloric effect which is induced by the variation of the system size.

The magnetocaloric effect is described by the so-called magnetic GP

$$
\Gamma_{\text {mag }}=-\frac{H}{T} \frac{\left.(\partial S / \partial H)\right|_{N, T, V}}{\left.(\partial S / \partial T)\right|_{N, H, V}}=\left.\frac{H}{T} \frac{\partial T}{\partial H}\right|_{S, N, V},
$$

where $S$ is the entropy. The magnetocaloric effect marks the change of temperature in response to an adiabatic change of $H$ [see (6)]. This feature has been used for the adiabatic demagnetization cooling $[23,24]$. We would like to mention that in contrast to some previous studies $[25,26]$, here we 
put the magnetic field $H$ in the numerator to make the GP dimensionless. Similarly, using the Maxwell relations and general thermal relations, we can obtain the expression of the magnetic GP in grand canonical ensemble [22]

$$
\Gamma_{\text {mag }}=-\frac{H}{T} \frac{\frac{\partial^{2} p}{\partial \mu^{2}} \frac{\partial^{2} p}{\partial H \partial T}-\frac{\partial^{2} p}{\partial \mu \partial H} \frac{\partial^{2} p}{\partial \mu \partial T}}{\frac{\partial^{2} p}{\partial \mu^{2}} \frac{\partial^{2} p}{\partial T^{2}}-\left(\frac{\partial^{2} p}{\partial \mu \partial T}\right)^{2}} .
$$

In ultracold atoms the interaction between two colliding atoms can be tuned by Feshbach resonances or confinementinduced resonances. The contact interaction can be regarded as a potential which changes the free energy in a grand canonical ensemble. The interacting caloric effect in quantum gases has been rarely studied [22,27]. Similarly, the interacting GP, describing the change of temperature in response to an adiabatic change of the interaction, can be given by

$$
\Gamma_{\text {int }}=-\frac{\left.c \frac{\partial S}{\partial c}\right|_{N, H, T, V}}{\left.T \frac{\partial S}{\partial T}\right|_{N, H, c, V}}=-\frac{c}{T} \frac{\frac{\partial^{2} p}{\partial \mu^{2}} \frac{\partial^{2} p}{\partial c \partial T}-\frac{\partial^{2} p}{\partial \mu \partial c} \frac{\partial^{2} p}{\partial \mu \partial T}}{\frac{\partial^{2} p}{\partial \mu^{2}} \frac{\partial^{2} p}{\partial T^{2}}-\left(\frac{\partial^{2} p}{\partial \mu \partial T}\right)^{2}},
$$

where $c$ denotes the interaction strength between atoms.

To summarize, the relations between the caloric effect and the GPs are given by

$$
\begin{gathered}
\left.\frac{\partial T}{\partial V}\right|_{S, N, H, c}=\frac{T}{V} \Gamma, \\
\left.\frac{\partial T}{\partial H}\right|_{S, N, V, c}=\frac{T}{H} \Gamma_{\mathrm{mag}}, \\
\left.\frac{\partial T}{\partial c}\right|_{S, N, V, H}=\frac{T}{c} \Gamma_{\mathrm{int}}
\end{gathered}
$$

that provide plausible experimental measurements of the GPs caused by the changes of the volume, magnetic field, and interaction, respectively. The given relation (7) marks the change of temperature in response to an adiabatic change of the interaction strength. Similar to the magnetic GP, the interacting GP is experimentally measurable and can be used to quantify the adiabatic refrigeration driven by interaction. We will study these three types of GPs for the one-dimensional (1D) Bose and Fermi gases in the following sections.

In this paper, we systematically study the GPs for 1D quantum gases using Bethe ansatz solutions. We analytically obtain the system size, magnetic field, and interaction-driven GPs for the Lieb-Liniger model [28] and the Yang-Gaudin model $[29,30]$.

The obtained GPs show volume, magnetic field, and interaction dependences of characteristic energy scales of the quantum gases and present the caloric effects resulting from the variations of these potentials. Our results confirm the identity among the three types of GPs [22] in 1D quantum systems:

$$
d \Gamma+2 \Gamma_{\text {mag }}+\Gamma_{\text {int }}=2 ;
$$

here $d=1$ is the dimensionality. We also derive the universal singular behaviors of the GPs at the quantum critical points in the attractive Yang-Gaudin model [29,30], driven either by the magnetic field or the interaction.

The divergent behaviors of the GPs near quantum critical points show the enhancement of the caloric effects at phase boundaries and help obtain the phase diagram and to realize quantum cooling.

The outline of this paper is as follows. In Sec. II, we study the GPs for the 1D Lieb-Liniger Bose gas. Analytical expression of the interacting GP is presented. In Sec. III, we present exact analytical expressions of the GPs for the Yang-Gaudin model in the fully polarized phase, fully paired phase, and the Fulde-Ferrell-Larkin-Ovchinnikov-(FFLO-) like pairing phase. We also studied the phase transitions of the model in terms of different GPs. We conclude with a brief summary in Sec. IV.

\section{GRÜNEISEN PARAMETER FOR THE LIEB-LINIGER MODEL}

The Lieb-Liniger model [28], which describes the 1D interacting bosons, is a prototypical Bethe ansatz solvable model [31]. It is one of the most extensively studied many-body systems in ultracold atoms. The Hamiltonian of the model is $(\hbar=2 m=1)$

$$
\hat{H}=-\sum_{i=1}^{N} \frac{\partial^{2}}{\partial x_{i}{ }^{2}}+2 c \sum_{i<j}^{N} \delta\left(x_{i}-x_{j}\right),
$$

where $N$ is the total number of spinless bosons constrained by periodic boundary conditions on a line of length $L$. For repulsive (attractive) contact interaction, the interaction strength $c>0(c<0)$. The coupling constant $c=-2 \hbar^{2} / m a_{1 \mathrm{D}}$ is determined by the $1 \mathrm{D}$ scattering length, given by $a_{1 \mathrm{D}}=$ $\left(-a_{\perp}^{2} / 2 a_{s}\right)\left[1-C\left(a_{s} / a_{\perp}\right)\right]$ [32-34]. Here the numerical constant $C \approx 1.4603$.

The Bethe ansatz wave function for the Lieb-Liniger model (9) is given by

$$
\begin{aligned}
\Psi= & \sum_{p}(-1)^{p}\left[\prod_{1 \leqslant i<j \leqslant N}\left(1+\frac{i k_{p_{j}}-i k_{p_{i}}}{c}\right)\right] \\
& \times \exp \left(\sum_{j=1}^{N} i k_{p_{j}} x_{j}\right),
\end{aligned}
$$

where $p$ stands for $N$ ! permutations of integers $1,2, \ldots, N$. The pseudomomenta $k_{j}$ satisfies the following BA equation:

$$
e^{i k_{j} L}=-\prod_{l=1}^{N} \frac{k_{j}-k_{l}+i c}{k_{j}-k_{l}-i c} .
$$

The solution to Eq. (11) provides complete spectra of the Lieb-Liniger model with $E=\sum_{j}^{N} k_{j}^{2}$.

In 1969, Yang and Yang [35] introduced the particle hole ensemble to describe the thermodynamics of the model in equilibrium, which is later called the thermodynamics Bethe ansatz (TBA) approach [36]. In terms of the dressed energy $\varepsilon(k)=T \ln \left[\rho^{h}(k) / \rho(k)\right]$ defined with respect to the quasimomentum $k$ at finite temperature $T$, the TBA equation is given by

$$
\varepsilon(k)=k^{2}-\mu-\frac{T c}{\pi} \int_{-\infty}^{+\infty} \frac{d q}{c^{2}+(k-q)^{2}} \ln \left(1+e^{-\varepsilon(q) / T}\right),
$$


where $\mu$ is the chemical potential. The dressed energy $\varepsilon(k)$ plays the role of excitation energy measured from the energy level $\varepsilon\left(k_{F}\right)=0$, where $k_{F}$ is the Fermi-like momentum. The pressure $p$ is given in terms of the temperature and chemical potential

$$
p=\frac{T}{2 \pi} \int_{-\infty}^{+\infty} \ln \left(1+e^{-\varepsilon(k) / T}\right) d k
$$

serving as the equation of states of the system.

For the strong repulsive interaction, the model (9) is called the Tonks-Girardeau (TG) gas [37]. In the TG limit, the Bose-Fermi mapping can be used to study the ground-state properties of the Bose gas, where the wave function of bosons can be written as the product of the sign function and the wave function of the noninteracting fermions. From the expressions of the GPs (1) and (4), by solving the TBA equation (12) we can numerically obtain the volume and interacting GPs (see Fig. 1). In Fig. 1(a) we see a subtle change of the GP as the interaction strength varies from zero to infinity. In these two limits, the scaling invariance leads to $\Gamma=2$ in 1D.

It is particularly interesting to investigate the interacting GP (4). In the strong interaction regime, $\gamma=c / n \gg 1$,
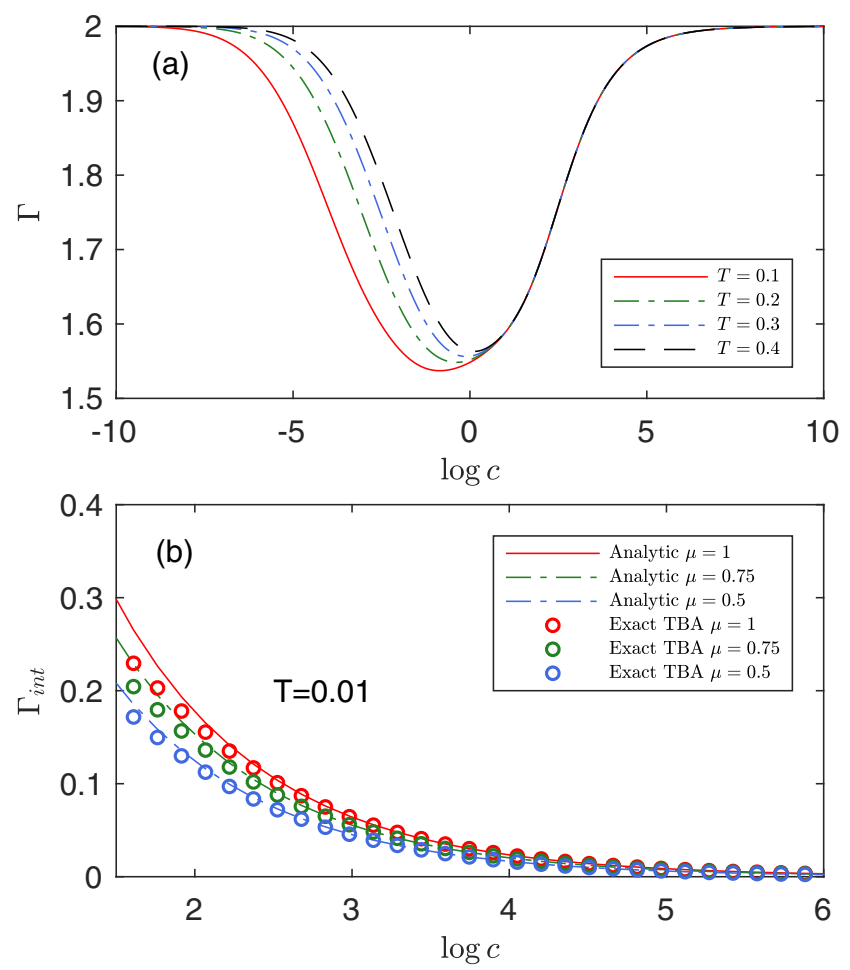

FIG. 1. (a) The Grüneisen parameter (1) versus interaction strength $\log c$ for the 1D Bose gas at different temperatures. Here the total density is fixed at $n=1$. In the weak coupling limit, the system behaves as a free Bose gas for which the GP $\Gamma=2$. Whereas in the strong coupling limit, it reduces to a fermionized Tonks-Girardeau gas with $\Gamma=2$, which shows a scaling invariant nature of the GP. (b) The interacting Grüneisen parameter (4) versus interaction strength $\log c$ for the strong coupling regime. Here we set $T=0.01$ and $\mu=1,0.75,0.5$. For a strong repulsion, we observe good agreement between the numerical (asterisks) and analytical (solid lines) results, Eq. (21).
$T / c^{2} \ll 1$, the TBA equation (12) can be expanded in the powers of $1 / c$ [up to the order of $O\left(1 / c^{3}\right)$ ] [38]:

$$
\varepsilon(k) \approx k^{2}-\mu-\frac{2 c}{c^{2}+k^{2}} p-\frac{1}{2 \sqrt{\pi} c^{3}} T^{5 / 2} \operatorname{Li}_{5 / 2}\left(-e^{A_{0} / T}\right),
$$

where $A_{0}=\mu+\frac{2 p}{c}-\frac{4 \mu^{5 / 2}}{15 \pi|c|^{3}}$ and $\operatorname{Li}_{s}(z)=\frac{1}{\Gamma(s)} \int_{0}^{+\infty} \frac{t^{s-1}}{e^{t} / z-1} d t$ denotes the polylogarithmic function. For simplicity, here we only consider the calculation up to the order of $1 / c^{2}$. Substituting Eq. (14) into Eq. (13), we obtain the pressure

$$
p=-\frac{1}{2 \sqrt{\pi}} T^{3 / 2} \operatorname{Li}_{3 / 2}\left(-e^{A / T}\right)
$$

with

$$
A=\mu+\frac{2}{c} p
$$

In the following calculation, we only consider the lowtemperature and strongly interacting regimes $T / c^{2} \ll 1, c \gg 1$ in order to obtain an explicit form of the Grüneisen parameter. In a canonical ensemble and in this low-temperature limit $T / c^{2} \ll 1$ with $c \gg 1$, we can easily calculate the pressure up to the order $O\left(T^{4}, 1 / c^{3}\right)$. Using the series expansion of the polylogarithmic function [39]

$$
\operatorname{Li}_{s}\left(-e^{x}\right)=-\frac{x^{s}}{\Gamma(s+1)}\left[1+\frac{\Gamma(s+1)}{6 \Gamma(s-1)}\left(\frac{\pi}{x}\right)^{2}+\cdots\right]
$$

in Eq. (15a), we have the following pressure:

$$
p=\frac{2}{3 \pi}\left(\mu+\frac{2}{c} p\right)^{3 / 2}+\frac{\pi T^{2}}{12}\left(\mu+\frac{2}{c} p\right)^{-1 / 2}+O\left(T^{4}, \frac{1}{c^{3}}\right)
$$

that essentially depends on the chemical potential and interaction. After a lengthy iteration with Eq. (17), we obtain a close form of the pressure up to the order $O\left(T^{4}, \frac{1}{c^{3}}\right)$ :

$$
\begin{aligned}
p \approx & \frac{2}{3 \pi} \mu^{3 / 2}+\frac{4}{3 \pi^{2} c} \mu^{2}+\frac{28}{9 \pi^{3} c^{2}} \mu^{5 / 2} \\
& +T^{2}\left(\frac{\pi}{12} \mu^{-1 / 2}+\frac{1}{9 c}+\frac{5}{18 \pi c^{2}} \mu^{1 / 2}\right) .
\end{aligned}
$$

Here we remark that for our purpose of calculating the GPs in the Luttinger liquid regions, we also need the leading order temperature contribution to the pressure. We separately considered the interaction and temperature orders in the above calculation.

In the low-temperature limit $T / c^{2} \ll 1$ with $c \gg 1$, the first- and second-order derivatives of pressure with respect to chemical potential can be obtained from Eq. (18):

$$
\begin{aligned}
\frac{\partial p}{\partial \mu} & =\frac{1}{\pi} \mu^{1 / 2}+\frac{8}{3 c \pi^{2}} \mu+\frac{70}{9 c^{2} \pi^{3}} \mu^{3 / 2}+O\left(T^{4}, 1 / c^{3}\right), \\
\frac{\partial^{2} p}{\partial \mu^{2}} & =\frac{1}{2 \pi} \mu^{-1 / 2}+\frac{8}{3 c \pi^{2}}+\frac{35}{3 c^{2} \pi^{3}} \mu^{1 / 2}+O\left(T^{4}, 1 / c^{3}\right) .
\end{aligned}
$$


Taking the partial derivative of Eq. (18) with respect to temperature and chemical potential, we have

$$
\begin{aligned}
\frac{\partial p}{\partial T} & =T\left[\frac{\pi}{6} \mu^{-1 / 2}+\frac{2}{9 c}+\frac{5}{9 \pi c^{2}} \mu^{1 / 2}+O\left(1 / c^{3}\right)\right], \\
\frac{\partial^{2} p}{\partial \mu \partial T} & =T\left[-\frac{\pi}{12} \mu^{-3 / 2}+\frac{5}{18 \pi c^{2}} \mu^{-1 / 2}+O\left(1 / c^{3}\right)\right], \\
\frac{\partial^{2} p}{\partial T^{2}} & =\frac{\pi}{6} \mu^{-1 / 2}+\frac{2}{9 c}+\frac{5}{9 \pi c^{2}} \mu^{1 / 2}+O\left(1 / c^{3}\right) .
\end{aligned}
$$

By substituting the above derivatives into the definition of GPs Eqs. (1) and (4), we obtain the explicit expression of the GPs:

$$
\Gamma=2-\frac{4 \sqrt{\mu}}{\pi c}-\frac{8 \mu}{3 \pi^{2} c^{2}}+O\left(\frac{1}{c^{3}}\right)
$$

and

$$
\Gamma_{\mathrm{int}}=\frac{4 \sqrt{\mu}}{\pi c}+\frac{8 \mu}{3 \pi^{2} c^{2}}+O\left(\frac{1}{c^{3}}\right) .
$$

Figure 1(b) shows that the analytical result (21) agrees well with the numerical calculation for the interacting GP. This clearly indicates a strong repulsion drives the system into an ideal gas with a scaling homogeneous spectrum in the limit $c \rightarrow \infty$. The two figures in Fig. 1 clearly indicate different features of the expansionary and interacting GPs. Our result further confirms the universal identity (8), namely,

$$
\Gamma+\Gamma_{\text {int }}=2 .
$$

This relation can be used to investigate interaction effect in quantum gases (see the analysis on a quantum heat engine in 1D Bose gas [27]).

\section{GRÜNEISEN PARAMETER FOR THE YANG-GAUDIN MODEL}

In this section we will study the magnetic and interacting GPs for the 1D Yang-Gaudin model with an attractive interaction $[29,30]$. The existence of a FFLO pairing state in the interacting Fermi gas has been predicted by exact solutions [40-42]. Recent breakthrough experiments on trapped ultracold fermionic atoms confined to 1D have provided a deep understanding of such a novel FFLO phase of the YangGaudin model [43] (see a review [44]). Here we will study the magnetic and interacting GPs throughout the full phase diagram of the model.

We will show a universal divergent feature of the GPs near quantum phase transitions. This feature can be used to probe quantum scaling, caloric effects, and quantum refrigeration in quantum many-body systems.

The Yang-Gaudin model $[29,30]$ described the 1D spin- $\frac{1}{2}$ Fermi gas with a $\delta$-function interaction. The Hamiltonian reads

$$
\hat{H}=-\sum_{i=1}^{N} \frac{\partial^{2}}{\partial x_{i}^{2}}+2 c \sum_{i<j}^{N} \delta\left(x_{i}-x_{j}\right)-\frac{1}{2} H\left(N_{\downarrow}-N_{\uparrow}\right)
$$

in which the terms are the kinetic energy, interaction energy, and Zeeman energy, respectively. Here $N$ is the total number of fermions in a length $L$ and $c<0$ indicates the contact attractive interaction. The Bethe ansatz (BA) equations were given by $[29,30]$

$$
\begin{aligned}
e^{i k_{j} L} & =\prod_{\alpha=1}^{M} \frac{k_{j}-\Lambda_{\alpha}+i c / 2}{k_{j}-\Lambda_{\alpha}-i c / 2}, \\
\prod_{j=1}^{N} \frac{\Lambda_{\alpha}-k_{j}+i c / 2}{\Lambda_{\alpha}-k_{j}-i c / 2} & =-\prod_{\beta=1}^{M} \frac{\Lambda_{\alpha}-\Lambda_{\beta}+i c}{\Lambda_{\alpha}-\Lambda_{\beta}-i c} .
\end{aligned}
$$

The energy of the system is given by $E=\sum_{j}^{N} k_{j}^{2}$. The model has spin population imbalance caused by a difference in the number of spin-up and spin-down atoms. The key feature of this ground-state phase diagram was experimentally confirmed by using finite temperature density profiles of trapped fermionic ${ }^{6} \mathrm{Li}$ atoms [43], where three quantum phasesfully paired state, partially polarized FFLO-like state, and fully polarized state-exist in a chemical potential-effective magnetic-field plane [40,41] (also see the phase diagram of the homogeneous Fermi gas $[19,42,44,45])$.

In order to build up the thermodynamic Bethe ansatz approach to the 1D attractive Fermi gas, we define the dressed energies $\epsilon^{b}(k)=T \ln \left[\rho_{2}^{h}(k) / \rho_{2}(k)\right]$ and $\epsilon^{u}(k)=$ $T \ln \left[\rho_{1}^{h}(k) / \rho_{1}(k)\right]$, corresponding to paired fermions and unpaired fermions in the grand canonical ensemble, respectively. According to the Yang-Yang method [35], by minimizing the free energy the TBA equations are given by $[36,44]$

$$
\begin{aligned}
\epsilon^{b}(k)= & 2\left(k^{2}-\mu-\frac{1}{4} c^{2}\right)+T a_{2} * \ln \left(1+e^{-\epsilon^{b}(k) / T}\right) \\
& +T a_{1} * \ln \left(1+e^{-\epsilon^{u}(k) / T}\right), \\
\epsilon^{u}(k)= & k^{2}-\mu-\frac{1}{2} H+T a_{1} * \ln \left(1+e^{-\epsilon^{b}(k) / T}\right) \\
& -T \sum_{l=1}^{\infty} a_{l} * \ln \left[1+\eta_{l}^{-1}(k)\right], \\
\ln \eta_{l}(\lambda)= & \frac{l H}{T}+a_{l} * \ln \left(1+e^{-\epsilon^{u}(\lambda) / T}\right) \\
& +\sum_{m=1}^{\infty} T_{l m} * \ln \left[1+\eta_{m}^{-1}(\lambda)\right],
\end{aligned}
$$

where $a_{n}(x)=\frac{1}{2 \pi} \frac{n|c|}{\left(\frac{n|c|}{2}\right)^{2}+x^{2}}$ and the function $T_{l m}$ is given in [44]. In the above equations, “ $*$ ” denotes convolution, namely, $a * f(x)=\int a(x-y) f(y) d y$. And $\eta_{l}(\lambda): \equiv \xi_{l}^{h}(\lambda) / \xi_{l}(\lambda)$ is the ratio between the hole density $\xi_{l}^{h}(\lambda)$ and the particle density $\xi_{l}(\lambda)$ of the length- $l$ strings, associated with the excitations of magnons. The pressure is given by

$$
\begin{aligned}
p= & \frac{T}{\pi} \int_{-\infty}^{\infty} d k \ln \left(1+e^{-\epsilon^{b}(k) / T}\right) \\
& +\frac{T}{2 \pi} \int_{-\infty}^{\infty} d k \ln \left(1+e^{-\epsilon^{u}(k) / T}\right) .
\end{aligned}
$$

The TBA Eq. (25) provide us with an analytical way to study the Grüneisen parameters.

By taking integration by part in the pressure (26), we then obtain the dimensionless pressure $\tilde{p}=\widetilde{p}^{(1)}+\widetilde{p}^{(2)}$ in terms of the leading terms of the dimensionless effective pressures 

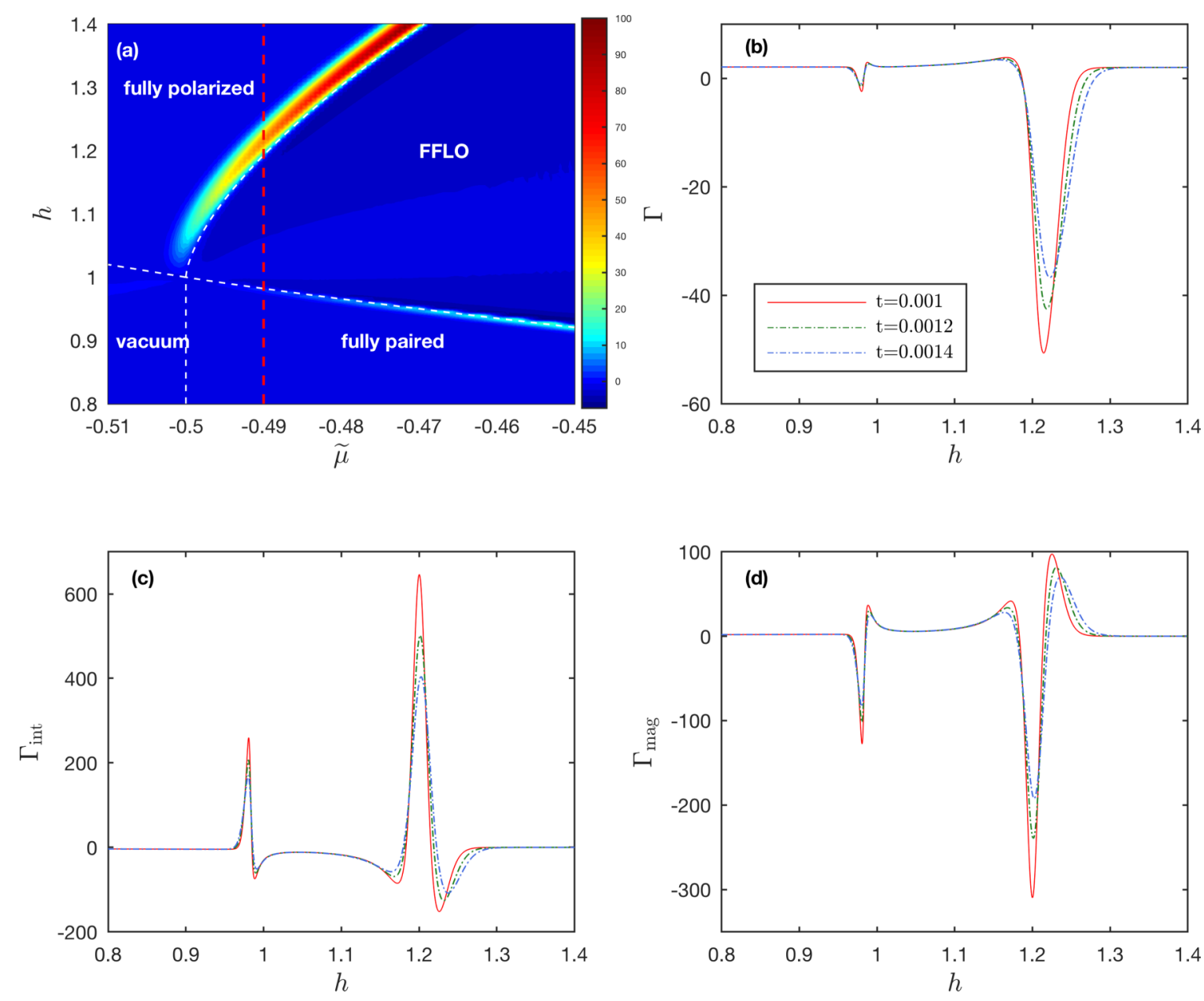

FIG. 2. (a) Phase diagram is mapped out from the contour plot of volume-driven GP, Eq. (1), at $t=0.001$. This expansionary GP has sudden enhancement near the phase boundaries, showing the quantum phase transitions between two different phases among fully paired, fully polarized, and FFLO-like phases. The white dashed lines denote the analytical zero-temperature critical fields (see [45]). (b)-(d) show the volume, interaction, and magnetic-field-driven GPs at $\tilde{\mu}=-0.49$ and $t=0.001,0.0012$, and 0.0014, respectively [see Eqs. (1), (4), and (3)]. These GPs characterize universal scaling behavior near a quantum phase transition.

$$
\widetilde{p}^{(r)}=\frac{p^{(r)}}{\frac{1}{2}|c|^{3}} \text { with } r=1,2, \text { i.e., }
$$

$$
\begin{aligned}
& \widetilde{p}^{(1)}=-\frac{1}{2 \sqrt{2 \pi}} t^{3 / 2} \operatorname{Li}_{3 / 2}\left(-e^{\widetilde{A}^{(1)} / t}\right), \\
& \widetilde{p}^{(2)}=-\frac{1}{2 \sqrt{\pi}} t^{3 / 2} \operatorname{Li}_{3 / 2}\left(-e^{\widetilde{A}^{(2)} / t}\right),
\end{aligned}
$$

where $\widetilde{A}^{(1)}=\widetilde{A}_{0}^{(1)}-2 \widetilde{p}^{(2)}$ with $\widetilde{A}_{0}^{(1)}=\widetilde{\mu}_{1}$ and $\widetilde{A}^{(2)}=\widetilde{A}_{0}^{(2)}-$ $\widetilde{p}^{(2)}-4 \widetilde{p}^{(1)}$ with $\widetilde{A}_{0}^{(2)}=\tilde{\mu}_{2}$. The superscripts (1) and (2) denote unpaired and paired fermions, respectively. Here we denoted the effective chemical potentials $\widetilde{\mu}_{1}=\widetilde{\mu}+\frac{1}{2} h$ and $\tilde{\mu}_{2}=2 \widetilde{\mu}+1$ for unpaired fermions and paired fermions, respectively. The dimensionless quantities used in the above equations are given by

$$
\begin{aligned}
\widetilde{A}^{(r)} & =\frac{A^{(r)}}{\frac{1}{2}|c|^{2}}, & & \tilde{\mu}=\frac{\mu}{\frac{1}{2}|c|^{2}}, \quad h=\frac{H}{\frac{1}{2}|c|^{2}}, \\
t & =\frac{T}{\frac{1}{2}|c|^{2}}, & \widetilde{p}^{(r)}=\frac{p^{(r)}}{\frac{1}{2}|c|^{3}} . &
\end{aligned}
$$

In Fig. 2(a), we contour plot the GP, Eq. (1), which shows a full phase diagram at low temperature. We observe the divergent behavior near the phase boundaries. At the phase boundaries, these GPs display universal divergent behaviors. Figures 2(b)-2(d) present different GPs for a fixed effective chemical potential $\tilde{\mu}=-0.49$ and temperature $t=0.001$, 0.0012 , and 0.0014 , respectively, showing sharp peaks near the phase transition between the FFLO-like phase and fully polarized phase as well as between the fully paired phase and the FFLO-like state. At these phase transitions, the GPs show strong caloric effect due to the accumulation of entropy, facilitating the realization of quantum cooling and quantum heat engines.

\section{A. The GPs for the fully polarized phase}

For a noninteracting system, scaling invariance gives rise to a constant value of the GP, i.e., $\Gamma=2 / d$; here $d$ is the dimensionality. For the Yang-Gaudin model, in the fully polarized phase, we have $\widetilde{A}^{(1)}>0$ and $\widetilde{A}^{(2)}<0$. Therefore only the pressure of the fully polarized fermions $\widetilde{p}^{(1)}$ contributes to the total pressure at low temperatures, 
namely,

$$
\begin{aligned}
& \widetilde{p}^{(1)}=-\frac{1}{2 \sqrt{2 \pi}} t^{3 / 2} \operatorname{Li}_{3 / 2}\left(-e^{\widetilde{A}^{(1)} / t}\right), \\
& \widetilde{A}^{(1)}=\widetilde{A}_{0}^{(1)} .
\end{aligned}
$$

Similar to the calculation for the Lieb-Liniger model, for calculating the GPs in the Luttinger liquid regions, we only consider the leading order temperature contribution to the first-order derivative of pressure with respect to the temperature. We may analytically obtain a series of all the derivatives of the pressure with respect to the temperature and chemical potential in the limits $t \ll \widetilde{A}_{0}^{(1)} \ll 1, t \ll 1$, and $\widetilde{\mu}_{1} \ll 1$ :

$$
\begin{aligned}
\tilde{p} & =\frac{\sqrt{2}}{3 \pi} \widetilde{\mu}_{1}^{3 / 2}+\frac{\pi}{12 \sqrt{2}} \tilde{\mu}_{1}^{-1 / 2} t^{2}+O\left(t^{4}\right), \\
\frac{\partial \widetilde{p}}{\partial \widetilde{\mu}} & =\frac{1}{\sqrt{2} \pi} \widetilde{\mu}_{1}^{1 / 2}+O\left(t^{2}\right), \\
\frac{\partial^{2} \widetilde{p}}{\partial \widetilde{\mu}^{2}} & =\frac{1}{2 \sqrt{2} \pi} \widetilde{\mu}_{1}^{-1 / 2}+O\left(t^{2}\right), \\
\frac{\partial \widetilde{p}}{\partial t} & =t\left(\frac{\pi}{6 \sqrt{2}} \widetilde{\mu}_{1}^{-1 / 2}\right)+O\left(t^{3}\right), \\
\frac{\partial^{2} \widetilde{p}}{\partial t^{2}} & =\frac{\pi}{6 \sqrt{2}} \widetilde{\mu}_{1}^{-1 / 2}+O\left(t^{2}\right), \\
\frac{\partial^{2} \widetilde{p}}{\partial \widetilde{\mu} \partial t} & =t\left(-\frac{\pi}{12 \sqrt{2}} \tilde{\mu}_{1}^{-3 / 2}\right)+O\left(t^{3}\right), \\
\frac{\partial^{2} \tilde{p}}{\partial h \partial t} & =-t\left(\frac{\pi}{24 \sqrt{2}} \tilde{\mu}_{1}^{-3 / 2}\right)+O\left(t^{3}\right), \\
\frac{\partial^{2} \tilde{p}}{\partial \tilde{\mu} \partial h} & =\frac{1}{4 \sqrt{2} \pi} \widetilde{\mu}_{1}^{-1 / 2}+O\left(t^{2}\right) .
\end{aligned}
$$

It is straightforward to obtain the Grüneisen parameter $\Gamma \approx 2$. While, with the help of the definition, Eq. (3), the magnetic GP is given by $\Gamma_{\text {mag }}=0$.

\section{B. The GPs for the fully paired phase}

In the fully paired phase, the system consists of pure pairs in the ground state. At low temperatures, i.e., $t \ll 1$, we have $\widetilde{A}^{(1)}<0$ and $\widetilde{A}^{(2)}>0$. Therefore, the effective pressure of paired fermions $\widetilde{p}^{(2)}$ mainly contributes to the total pressure. It follows that

$$
\begin{aligned}
& \widetilde{p}^{(2)}=-\frac{1}{2 \sqrt{\pi}} t^{3 / 2} \operatorname{Li}_{3 / 2}\left(-e^{\widetilde{A}^{(2)} / t}\right), \\
& \widetilde{A}^{(2)}=\widetilde{A}_{0}^{(2)}-\widetilde{p}^{(2)} .
\end{aligned}
$$

Similarly, in the limits $t \ll \widetilde{A}_{0}^{(2)} \ll 1, t \ll 1$, and $\widetilde{\mu}_{2} \ll 1$, we separately consider different interaction and temperature orders and obtain the pressure and the leading order of the derivatives with respect to chemical potential and temperature:

$$
\begin{aligned}
\tilde{p}= & \frac{2}{3 \pi} \widetilde{\mu}_{2}^{3 / 2}-\frac{2}{3 \pi^{2}} \widetilde{\mu}_{2}^{2}+\frac{7}{9 \pi^{3}} \widetilde{\mu}_{2}^{5 / 2} \\
& +t^{2}\left(\frac{\pi}{12} \widetilde{\mu}_{2}^{-1 / 2}-\frac{1}{18}+\frac{5}{72 \pi} \widetilde{\mu}_{2}^{1 / 2}\right)+O\left(t^{4}, \tilde{\mu}_{2}^{3}\right),
\end{aligned}
$$

$$
\begin{aligned}
& \frac{\partial \widetilde{p}}{\partial \widetilde{\mu}}=\frac{2}{\pi} \widetilde{\mu}_{2}^{1 / 2}-\frac{8}{3 \pi^{2}} \widetilde{\mu}_{2}+\frac{35}{9 \pi^{3}} \widetilde{\mu}_{2}^{3 / 2}+O\left(t^{2}, \widetilde{\mu}_{2}^{2}\right), \\
& \frac{\partial^{2} \widetilde{p}}{\partial \widetilde{\mu}^{2}}=\frac{2}{\pi} \widetilde{\mu}_{2}^{-1 / 2}-\frac{16}{3 \pi^{2}}+\frac{35}{3 \pi^{3}} \widetilde{\mu}_{2}^{1 / 2}+O\left(t^{2}, \widetilde{\mu}_{2}\right), \\
& \frac{\partial \widetilde{p}}{\partial t}=\frac{\pi t}{6}\left[\tilde{\mu}_{2}^{-1 / 2}-\frac{2}{3 \pi}+\frac{5}{6 \pi^{2}} \tilde{\mu}_{2}^{1 / 2}+O\left(\tilde{\mu}_{2}\right)\right], \\
& \frac{\partial^{2} \widetilde{p}}{\partial t^{2}}=\frac{\pi}{6}\left[\widetilde{\mu}_{2}^{-1 / 2}-\frac{2}{3 \pi}+\frac{5}{6 \pi^{2}} \widetilde{\mu}_{2}^{1 / 2}+O\left(\tilde{\mu}_{2}\right)\right], \\
& \frac{\partial^{2} \tilde{p}}{\partial \widetilde{\mu} \partial t}=t\left[-\frac{\pi}{6} \widetilde{\mu}_{2}^{-3 / 2}+\frac{5}{36 \pi} \widetilde{\mu}_{2}^{-1 / 2}+O(1)\right], \\
& \frac{\partial^{2} \widetilde{p}}{\partial h \partial t}=0, \\
& \frac{\partial^{2} \tilde{p}}{\partial \widetilde{\mu} \partial h}=0 .
\end{aligned}
$$

Substituting these derivatives into Eq. (1), we obtain the expansionary GP

$$
\Gamma=2+\frac{2 \sqrt{\tilde{\mu}_{2}}}{\pi}-\frac{2 \tilde{\mu}_{2}}{3 \pi^{2}} .
$$

The last two terms in this expression indicate the interaction effect of the spin singlet interaction. In fact, for the fully paired phase, the strongly attractive Fermi gas without polarization can be regarded as the super Tonks-Girardeau gas composed of bosonic Fermi pairs with a weakly attractive pair-pair interaction. By a transformation $c \rightarrow-c, \mu_{2}=2 \mu$, we prove that Eqs. (20) and (31) are equivalent, i.e.,

$$
\Gamma=2-\frac{4 \sqrt{\mu}}{\pi c}-\frac{8 \mu}{3 \pi^{2} c^{2}} .
$$

Thus we see that the GP for the attractive fermionic pairs is equivalent to that of the hard-core bosons [see (20)]. In addition, the magnetic GP, Eq. (3), can be calculated as $\Gamma_{\text {mag }}=0$.

\section{The GPs for the FFLO-like pairing phase}

In the FFLO-like pairing phase, the system exhibits two states: Paired and unpaired fermions. The spectra of the system are subtly influenced by the system size, interaction strength, and magnetic field. In this phase, $\widetilde{A}^{(1)}>0$ and $\widetilde{A}^{(2)}>0$. Therefore we need to solve the two branches of the TBA equations (25). Without losing generality, in order to capture the main features of the GPs at low temperatures, here we only consider the leading order of the effective pressures. By iteration of the TBA equations, the pressures of excess fermions and bound pairs are respectively given by

$$
\begin{aligned}
& \widetilde{p}^{(1)}=-\frac{1}{2 \sqrt{2 \pi}} t^{3 / 2} \operatorname{Li}_{3 / 2}\left(-e^{\widetilde{A}^{(1)} / t}\right), \\
& \widetilde{p}^{(2)}=-\frac{1}{2 \sqrt{\pi}} t^{3 / 2} \operatorname{Li}_{3 / 2}\left(-e^{\widetilde{A}^{(2)} / t}\right), \\
& \widetilde{A}^{(1)}=\widetilde{\mu}_{1}-2 \widetilde{p}^{(2)}, \\
& \widetilde{A}^{(2)}=\widetilde{\mu}_{2}-\widetilde{p}^{(2)}-4 \widetilde{p}^{(1)} .
\end{aligned}
$$

Similarly, in the limits $t \ll \widetilde{A}_{0}^{(1,2)} \ll 1, t \ll 1$, and $\widetilde{\mu}_{1,2} \ll 1$, we separately consider different interaction and temperature 
orders. After a lengthy iteration, we calculate the pressure of excess fermions and bound pairs up to the order $O\left(T^{4}, \tilde{\mu}_{1,2}^{5 / 2}\right)$, respectively,

$$
\begin{gathered}
\widetilde{p}^{(1)}=\frac{\sqrt{2}}{3 \pi} \widetilde{\mu}_{1}^{3 / 2}-\frac{2 \sqrt{2}}{3 \pi^{2}} \widetilde{\mu}_{1}^{1 / 2} \widetilde{\mu}_{2}^{3 / 2}+t^{2}\left(\frac{\pi}{12 \sqrt{2}} \widetilde{\mu}_{1}^{-1 / 2}-\sqrt{2} / 12 \widetilde{\mu}_{1}^{1 / 2} \widetilde{\mu}_{2}^{-1 / 2}+\frac{1}{18 \sqrt{2}} \widetilde{\mu}_{1}^{-3 / 2} \widetilde{\mu}_{2}^{3 / 2}\right)+O\left(t^{4}, \widetilde{\mu}_{1,2}^{5 / 2}\right), \\
\widetilde{p}^{(2)}=\frac{2}{3 \pi} \widetilde{\mu}_{2}^{3 / 2}-\frac{2}{3 \pi^{2}} \widetilde{\mu}_{2}^{2}-\frac{4 \sqrt{2}}{3 \pi^{2}} \widetilde{\mu}_{1}^{3 / 2} \widetilde{\mu}_{2}^{1 / 2}+t^{2}\left(\frac{\pi}{12} \widetilde{\mu}_{2}^{-1 / 2}-\frac{1}{18}-\frac{1}{3 \sqrt{2}} \widetilde{\mu}_{1}^{-1 / 2} \widetilde{\mu}_{2}^{1 / 2}+\frac{\sqrt{2}}{18} \widetilde{\mu}_{1}^{3 / 2} \widetilde{\mu}_{2}^{-3 / 2}\right)+O\left(t^{4}, \widetilde{\mu}_{1,2}^{5 / 2}\right) .
\end{gathered}
$$

Then we obtain the leading terms of the derivatives of the pressure:

$$
\begin{aligned}
\frac{\partial \tilde{p}}{\partial \widetilde{\mu}} & =\frac{1}{\sqrt{2} \pi} \tilde{\mu}_{1}^{1 / 2}+\frac{2}{\pi} \widetilde{\mu}_{2}^{1 / 2}+O\left(t^{2}, \tilde{\mu}_{1,2}\right), \\
\frac{\partial \tilde{p}}{\partial t} & =t\left(\frac{\pi}{6 \sqrt{2}} \tilde{\mu}_{1}^{-1 / 2}+\frac{\pi}{6} \tilde{\mu}_{2}^{-1 / 2}\right)+O\left(t, \tilde{\mu}_{1,2}^{1 / 2}\right), \\
\frac{\partial^{2} \tilde{p}}{\partial \widetilde{\mu}^{2}} & =\frac{1}{2 \sqrt{2} \pi} \tilde{\mu}_{1}^{-1 / 2}+\frac{2}{\pi} \tilde{\mu}_{2}^{-1 / 2}+O\left(t^{2}, \tilde{\mu}_{1,2}^{1 / 2}\right), \\
\frac{\partial^{2} \tilde{p}}{\partial t^{2}} & =\frac{\pi}{6 \sqrt{2}} \tilde{\mu}_{1}^{-1 / 2}+\frac{\pi}{6} \tilde{\mu}_{2}^{-1 / 2}+O\left(1, \tilde{\mu}_{1,2}^{1 / 2}\right), \\
\frac{\partial^{2} \tilde{p}}{\partial \widetilde{\mu} \partial t} & =t\left(-\frac{\pi}{12 \sqrt{2}} \tilde{\mu}_{1}^{-3 / 2}-\frac{\pi}{6} \tilde{\mu}_{2}^{-3 / 2}\right)+O\left(t, \tilde{\mu}_{1,2}^{1 / 2}\right), \\
\frac{\partial^{2} \tilde{p}}{\partial h \partial t} & =-\frac{\pi}{24 \sqrt{2}} \tilde{\mu}_{1}^{-3 / 2} t+O\left(t, \tilde{\mu}_{1,2}^{1 / 2}\right), \\
\frac{\partial^{2} \tilde{p}}{\partial \tilde{\mu} \partial h} & =\frac{1}{4 \sqrt{2} \pi} \tilde{\mu}_{1}^{-1 / 2}+O\left(t^{2}, \tilde{\mu}_{1,2}^{1 / 2}\right) .
\end{aligned}
$$

Thus by definition Eq. (1), the Grüneisen parameter for the FFLO-like phase is given by

$$
\Gamma=2+\frac{\frac{1}{\sqrt{2}}\left(\lambda-\frac{2}{\lambda}\right)\left(\lambda-\frac{1}{2 \lambda}\right)}{\frac{1}{4 \lambda}+2 \lambda+\frac{5}{2 \sqrt{2}}},
$$

where the $\lambda$ is defined by $\lambda=\sqrt{\widetilde{\mu}_{1} / \widetilde{\mu}_{2}}$, which is related to the ratio between the densities of unpaired fermions and bound pairs, i.e., $\tilde{\mu}_{1} / \tilde{\mu}_{2} \sim n_{1} / n_{2}$. This expression is valid only for the strong-coupling limit that the rescaled temperature $t$ is much less than unity. Here the effective chemical potentials of unpaired fermions and paired fermions are given by $\widetilde{\mu}_{1}=\widetilde{\mu}+$ $\frac{1}{2} h$ and $\tilde{\mu}_{2}=2 \tilde{\mu}+1$, respectively. In Fig. 3(a), we observe good agreement between the numerical result obtained from the TBA equations (25) and the analytical results, Eq. (37), at different effective chemical potentials. For small and large values of the $\lambda$, the GP increases near the phase boundaries.

Similarly, at low temperatures, $t \ll 1$, we obtain the magnetic Grüneisen parameter from Eq. (3):

$$
\Gamma_{\text {mag }}=-\frac{h}{\widetilde{\mu}_{2}} \frac{1-\frac{2}{\lambda^{2}}}{8 \sqrt{2} \lambda+\frac{\sqrt{2}}{\lambda}+10} .
$$

Here $h=2 \tilde{\mu}_{2} \lambda^{2}-\tilde{\mu}_{2}+1$. The magnetic GP is also a dimensionless parameter and related to the magnetocaloric effect (see Fig. 3). In this figure, we also observe good agreement between numerical and analytical results of the GPs at low temperature $T=1.0 \times 10^{-5}$ in the strong coupling $|c|=10$ and for different total chemical potentials. From Eq. (38), we observe that the magnetic GP is equal to zero when $\lambda^{2}=2$. This point corresponds to the fact that effective chemical potentials of excess fermions and bound pairs are equal, called a critical polarization.

Moreover, with the help of the relation Eq. (8), we can further obtain the interaction GP through the relation $\Gamma_{\text {int }}=$ $2-\Gamma-2 \Gamma_{\mathrm{mag}}$. Substituting Eqs. (37) and (38) into Eq. (8), we have

$$
\Gamma_{\text {int }}=\frac{\left(\frac{1}{\widetilde{\mu}_{2}}-\lambda^{2}\right)\left(1-\frac{2}{\lambda^{2}}\right)}{\frac{1}{\sqrt{2} \lambda}+4 \sqrt{2} \lambda+5} .
$$

In Fig. 3(c), we observe that the result Eq. (39) is in good agreement with the numerical result obtained from the TBA equations (25) according to the definition Eq. (4). This agreement further confirms the identity Eq. (8) among the three
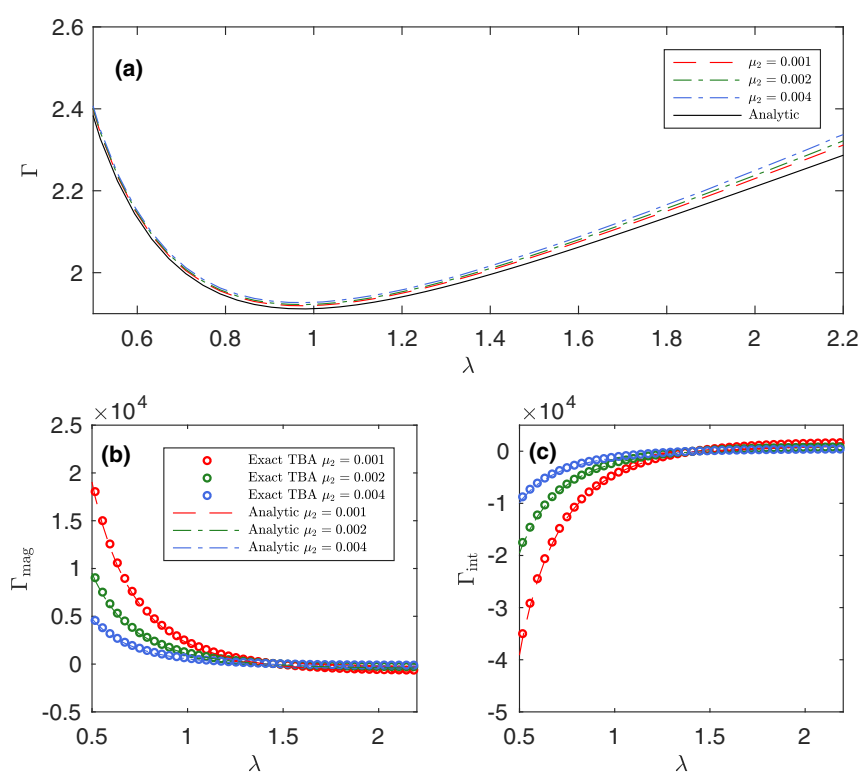

FIG. 3. (a) The Grüneisen parameter, Eq. (1), vs the effective chemical potential ratio $\lambda$ for the FFLO-like phase. Dashed lines show the numerical result from Eq. (1) and the TBA equations (25). The black solid line presents the result of Eq. (37) having good agreement with the numerical result. (b) The magnetic Grüneisen parameter, Eq. (3), vs the effective chemical potential ratio $\lambda$ for the FFLO-like phase. (c) The interaction Grüneisen parameter, Eq. (4), vs the effective chemical potential ratio $\lambda$ for the FFLO-like phase. A good agreement between the numerical (symbols) and analytical (dashed lines) results in the low-density and low-temperature limits. All shown data have been set for low-temperature $T=1.0 \times 10^{-5}$, $|c|=10$ in the strong-coupling region and the chemical potential $\mu_{2}=0.001,0.002$, and 0.004 , respectively. 


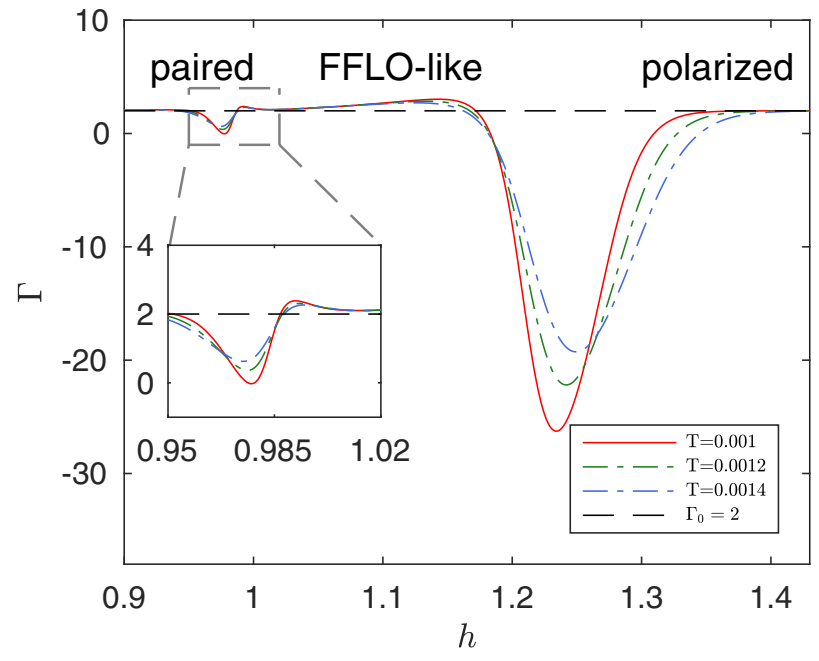

FIG. 4. The Grüneisen parameter vs magnetic field for fixed rescaled chemical potential $\mu=-0.245$. By definition (1), the numerical result of the GP is obtained from the TBA equation (25). For the strong-coupling region, we observe that the GP is very close to the line $\Gamma=2$ in the fully polarized phase and in the fully paired phase due to scaling invariant nature. It shows the universal singular behavior near the quantum phase transitions.

types of GPs. The interaction GP shows an interacting caloric effect which looks opposite to the magnetocaloric effect. We also notice the zero point of the interacting GP at $\lambda^{2}=2$ [see Fig. 3(c)]. This point is very special because the GP is a constant $\Gamma=2$, i.e., the energy spectrum has a scaling invariant. This opens a way to realize adiabatic interacting quantum cooling in quantum gases of ultracold atoms (also see cooling and thermometry of atomic Fermi gases [46]).

\section{The Grüneisen parameters at quantum criticality}

Quantum phase transitions occur in the 1D attractive Fermi gas at zero temperature by varying the external fields such as chemical potential and magnetic field. In Fig. 2, we find that the Grüneisen parameters display singular behavior near the quantum critical points. Such kind of singular behavior has been used to signify quantum phase transition [13,21]. Hence, the GPs can be regarded as a good probe of quantum criticality and associated universal scaling. An important observation of the GPs is that both the magnetic field and interacting GPs characterize the caloric effects for quantum cooling and heat engines. Here we further study the GPs for the 1D attractive Yang-Gaudin model, in which the potentials drive the system into four different phases, i.e., vacuum, fully paired, FFLOlike pairing (partially polarized), and fully polarized phases [see Fig. 2(a)].

Figures 4 and 5 show the magnetic field and interacting GPs at different temperatures, respectively. In Fig. 4, we fixed the chemical potential at $\mu=-0.245$. The long black dashed line stands for the constant value $\Gamma=2$ in the two figures. We observe that GPs are very close to the value of $\Gamma=2$ in the fully polarized phase and in the fully paired phase, where for $t \ll 1$ the system has scaling invariant nature. However, near the phase boundaries, they are suddenly enhanced and show a universal divergent nature. This divergent behavior elegantly

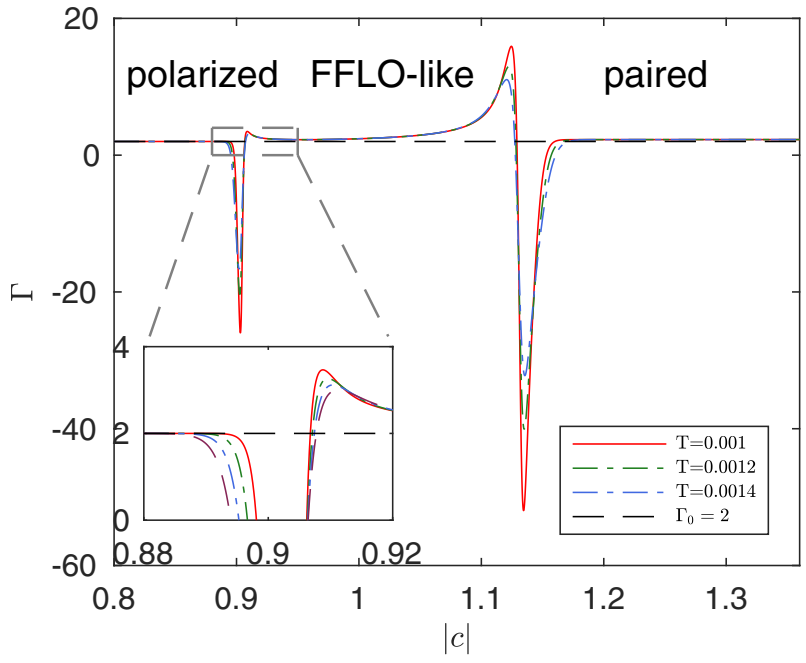

FIG. 5. The Grüneisen parameter vs interaction strength $c$ for fixed chemical potential $\mu=-0.2$ and magnetic field $H=0.5$. By definition (1), the numerical result of the GP is obtained from the TBA equation (25). For the strong-coupling region, we observe that like Fig. 4, the GP is very close to the line $\Gamma=2$ in the fully polarized phase and in the fully paired phase due to scaling invariant nature. It shows the universal singular behavior near the quantum phase transitions.

characterizes the critical phenomenon of the model, i.e., thermodynamic quantities display universal quantum scalings in the vicinities of critical points as temperature approaches to zero. In the following we will discuss the scaling functions of the GPs near the two nontrivial phase transitions: From the fully paired phase to the FFLO-like phase and from the FFLO-like state to the fully polarized phase. The calculation is presented in the Appendix.

Phase transition P-FFLO. Firstly, we discuss the GPs near the phase boundary between the fully paired phase and the FFLO-like state. We assume that the phase transition from the fully paired phase to the FFLO-like phase is driven by the effective chemical potential $\tilde{\mu}$. The phase boundary P-FFLO for strong coupling is determined by $\widetilde{\mu}_{c 1}=-\frac{h}{2}+$ $\frac{4}{3 \pi}(1-h)^{3 / 2}$, where $0<1-h \ll 1$. Up to the second order of $(1-h)$, we get the scaling functions of the thermodynamics quantities:

$$
\begin{gathered}
\tilde{n}=\frac{\partial \widetilde{p}}{\partial \widetilde{\mu}}=\frac{2}{\pi} \sqrt{b}-\frac{1}{2 \sqrt{2 \pi}} t^{1 / 2} \mathcal{R}_{0}\left(\frac{\widetilde{A_{1}}}{t}\right), \\
\tilde{s}=\frac{\partial \widetilde{p}}{\partial t}=\frac{1}{2 \sqrt{2 \pi}} t^{1 / 2} \mathcal{R}_{1}\left(\frac{\widetilde{A_{1}}}{t}\right), \\
\widetilde{m}=\frac{\partial^{2} \widetilde{p}}{\partial \widetilde{\mu}^{2}}=\frac{2}{\pi \sqrt{b}}+\frac{1}{2 \sqrt{2 \pi}} t^{-1 / 2} \mathcal{R}_{2}\left(\frac{\widetilde{A_{1}}}{t}\right), \\
\widetilde{c_{v} / t}=\frac{\partial^{2} \widetilde{p}}{\partial t^{2}}=-\frac{1}{2 \sqrt{2 \pi}} t^{-1 / 2} \mathcal{R}_{3}\left(\frac{\widetilde{A_{1}}}{t}\right), \\
\frac{\partial^{2} \tilde{p}}{\partial \widetilde{\mu} \partial t}=\frac{1}{2 \sqrt{2 \pi}} t^{-1 / 2} \mathcal{R}_{4}\left(\frac{\widetilde{A_{1}}}{t}\right),
\end{gathered}
$$



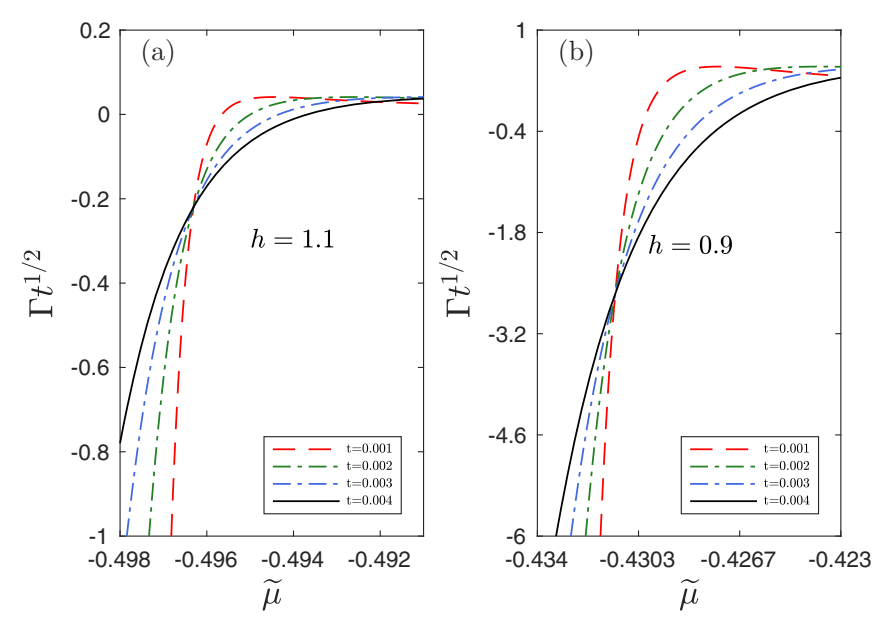

FIG. 6. Universal scaling behavior of the GP vs the rescaled chemical potential $\tilde{\mu}$ at different temperatures $t=0.001,0.002$, 0.003 , and 0.004 . The GP is calculated by the TBA equation (25). The intersections in the left and right panels give the phase boundaries of the F-FFLO and P-FFLO transitions, respectively.

$$
\begin{aligned}
\frac{\partial^{2} \tilde{p}}{\partial h \partial t} & =\frac{1}{4 \sqrt{2 \pi}} t^{-1 / 2} \mathcal{R}_{4}\left(\frac{\widetilde{A}_{1}}{t}\right), \\
\frac{\partial^{2} \widetilde{p}}{\partial \widetilde{\mu} \partial h} & =\frac{1}{4 \sqrt{2 \pi}} t^{-1 / 2} \mathcal{R}_{2}\left(\frac{\widetilde{A_{1}}}{t}\right),
\end{aligned}
$$

where $\tilde{A}_{1}=\left(\tilde{\mu}-\tilde{\mu}_{c 1}\right)$ and $b=(1-h)\left(1+\frac{2}{\pi} \sqrt{1-h}\right)$. While the analytic functions $\mathcal{R}_{i}(i=0,1,2,3,4)$ are given by

$$
\begin{aligned}
& \mathcal{R}_{0}(x)=\operatorname{Li}_{1 / 2}\left(-e^{x}\right), \\
& \mathcal{R}_{1}(x)=-\frac{3}{2} \operatorname{Li}_{3 / 2}\left(-e^{x}\right)+x \operatorname{Li}_{1 / 2}\left(-e^{x}\right), \\
& \mathcal{R}_{2}(x)=-\mathrm{Li}_{-1 / 2}\left(-e^{x}\right), \\
& \mathcal{R}_{3}(x)=\frac{3}{4} \operatorname{Li}_{3 / 2}\left(-e^{x}\right)-x \operatorname{Li}_{1 / 2}\left(-e^{x}\right)+x^{2} \operatorname{Li}_{-1 / 2}\left(-e^{x}\right), \\
& \mathcal{R}_{4}(x)=-\frac{1}{2} \operatorname{Li}_{1 / 2}\left(-e^{x}\right)+x \operatorname{Li}_{-1 / 2}\left(-e^{x}\right) .
\end{aligned}
$$

By the definition of the GP Eq. (1), we obtain an explicit form

$$
\Gamma=\frac{4 \sqrt{2 b}}{\sqrt{\pi}} t^{-1 / 2} \mathcal{G}\left(\frac{\tilde{\mu}-\tilde{\mu}_{c 1}}{t}\right),
$$

where $b=(1-h)\left(1+\frac{2}{\pi} \sqrt{1-h}\right)$ and

$$
\mathcal{G}(x)=\frac{\mathcal{R}_{4}}{\mathcal{R}_{2} \mathcal{R}_{3}+\mathcal{R}_{4} \mathcal{R}_{4}} .
$$

For the low-temperature region, i.e., $t \ll 1$, the GP Eq. (1) has a universal scaling $t^{-1 / 2}$ for the phase transition P-FFLO.

In Fig. 6(b), we demonstrate this universal scaling behavior for the phase transition P-FFLO.

Similarly, by the definition of the magnetic GP Eq. (3), we obtain the scaling function of the magnetic GP:

$$
\Gamma_{\text {mag }}=h \frac{2 \sqrt{2}}{\sqrt{\pi} \sqrt{b}} t^{-1 / 2} \mathcal{G}\left(\frac{\tilde{\mu}-\tilde{\mu}_{c 1}}{t}\right) .
$$

This shows a similar universal scaling behavior of the magnetic GP, $\Gamma_{\text {mag }} \propto t^{-1 / 2}$.
Regarding the scaling property of the pressure $p=$ $\frac{c^{3}}{2} \widetilde{p}\left(\frac{\mu}{c^{2} / 2}, \frac{T}{c^{2} / 2}, \frac{H}{c^{2} / 2}\right)$, we can obtain

$$
\begin{aligned}
\frac{\partial^{2} p}{\partial c \partial T} & =\frac{1}{c}\left[\frac{\partial p}{\partial T}-2 \mu \frac{\partial^{2} p}{\partial \mu \partial T}-2 T \frac{\partial^{2} p}{\partial T^{2}}-2 H \frac{\partial^{2} p}{\partial H \partial T}\right], \\
\frac{\partial^{2} p}{\partial c \partial \mu} & =\frac{1}{c}\left[\frac{\partial p}{\partial \mu}-2 \mu \frac{\partial^{2} p}{\partial \mu^{2}}-2 T \frac{\partial^{2} p}{\partial T \partial \mu}-2 H \frac{\partial^{2} p}{\partial H \partial \mu}\right] .
\end{aligned}
$$

It follows that

$$
\begin{aligned}
\frac{\partial^{2} p}{\partial c \partial T}= & \frac{\sqrt{2}}{c}\left(-\mu \frac{1}{\sqrt{2 \pi}}-H \frac{1}{2 \sqrt{2 \pi}}\right) T^{-\frac{1}{2}} \mathcal{R}_{4}\left(\frac{A_{1}}{T}\right), \\
\frac{\partial^{2} p}{\partial c \partial \mu}= & \frac{2}{\pi} \sqrt{b}-\frac{2}{c^{2}} \mu \frac{4}{\pi \sqrt{b}}-\frac{\sqrt{2}}{c} \mu \frac{1}{\sqrt{2 \pi}} T^{-1 / 2} \mathcal{R}_{2}\left(\frac{A_{1}}{T}\right) \\
& -\frac{\sqrt{2}}{c} H \frac{1}{2 \sqrt{2 \pi}} T^{-1 / 2} \mathcal{R}_{2}\left(\frac{A_{1}}{T}\right) .
\end{aligned}
$$

From the definition of the interaction GP Eq. (4), we have an explicit form of scaling function:

$$
\begin{aligned}
\Gamma_{\mathrm{int}}= & -\frac{4 \sqrt{2} \sqrt{b}}{\sqrt{\pi}} t^{-1 / 2} \mathcal{G}\left(\frac{\tilde{\mu}-\tilde{\mu}_{c 1}}{t}\right) \\
& -h \frac{4 \sqrt{2}}{\sqrt{\pi} \sqrt{b}} t^{-1 / 2} \mathcal{G}\left(\frac{\tilde{\mu}-\tilde{\mu}_{c 1}}{t}\right) .
\end{aligned}
$$

It can be seen from the above scaling functions that three types of GPs satisfy the identity

$$
\Gamma=2-2 \Gamma_{\text {mag }}-\Gamma_{\text {int }} .
$$

Phase transition FFLO-F. We now consider the GPs near the phase transition from the FFLO-like pairing phase to the fully polarized phase. This phase transition occurs by the variation of the chemical potential $\tilde{\mu}$ too. The critical phase boundary is determined by $\widetilde{\mu}_{c 2}=-\frac{1}{2}+\frac{1}{3 \pi}(h-1)^{3 / 2}$; here $h>1$. We expand it to the second order of $(h-1)$; we obtained the scaling functions of the thermodynamics quantities:

$$
\begin{aligned}
\frac{\partial \tilde{p}}{\partial \tilde{\mu}} & =\frac{\sqrt{a}}{2 \pi}-\frac{1}{\sqrt{\pi}} t^{1 / 2} \mathcal{R}_{0}\left(\frac{\widetilde{A_{2}}}{t}\right), \\
\frac{\partial \tilde{p}}{\partial t} & =\frac{1}{2 \sqrt{\pi}} t^{1 / 2} \mathcal{R}_{1}\left(\frac{\widetilde{A_{2}}}{t}\right), \\
\frac{\partial^{2} \widetilde{p}}{\partial \widetilde{\mu}^{2}} & =\frac{1}{2 \pi \sqrt{a}}+\frac{2}{\sqrt{\pi}} t^{-1 / 2} \mathcal{R}_{2}\left(\frac{\widetilde{A_{2}}}{t}\right), \\
\frac{\partial^{2} \widetilde{p}}{\partial t^{2}} & =-\frac{1}{2 \sqrt{\pi}} t^{-1 / 2} \mathcal{R}_{3}\left(\frac{\widetilde{A_{2}}}{t}\right), \\
\frac{\partial^{2} \widetilde{p}}{\partial \widetilde{\mu} \partial t} & =\frac{1}{\sqrt{\pi}} t^{-1 / 2} \mathcal{R}_{4}\left(\frac{\widetilde{A}_{2}}{t}\right), \\
\frac{\partial^{2} \widetilde{p}}{\partial h \partial t} & =-\frac{\sqrt{a}}{2 \pi{ }^{3 / 2}} t^{-1 / 2} \mathcal{R}_{4}\left(\frac{\widetilde{A}_{2}}{t}\right), \\
\frac{\partial^{2} \widetilde{p}}{\partial \tilde{\mu} \partial h} & =\frac{1}{4 \pi} \frac{1}{\sqrt{a}}-\frac{\sqrt{a}}{\pi^{3 / 2}} t^{-1 / 2} \mathcal{R}_{2}\left(\frac{\widetilde{A_{2}}}{t}\right),
\end{aligned}
$$


where by definition $\widetilde{A}_{2}=2\left(\tilde{\mu}-\widetilde{\mu}_{c 2}\right)$ and $\widetilde{\mu}_{c 2}$ denotes the critical chemical potential.

In the above equation $a=(h-1)\left(1+\frac{2}{3 \pi} \sqrt{h-1}\right)$, which is almost a constant. The scaling function of the GP at this phase boundary is given by

$$
\Gamma=\frac{\sqrt{a}}{2 \sqrt{\pi}} t^{-1 / 2} \mathcal{G}\left(\frac{2\left(\tilde{\mu}-\tilde{\mu}_{c 2}\right)}{t}\right) .
$$

The GP near the phase transition shows the same scaling behavior $\Gamma \propto t^{-1 / 2}$ as that for the phase transition P-FFLO. Figure 6(a) shows the scaling behavior near the phase transition FFLO-F. Moreover, the scaling function of the magnetic GP near the phase transition FFLO-F is given by

$$
\Gamma_{\text {mag }}=-h \frac{\frac{1}{\pi}+\frac{1}{\sqrt{a}}}{4 \sqrt{\pi}} t^{-1 / 2} \mathcal{G}\left(\frac{2\left(\tilde{\mu}-\tilde{\mu}_{c 2}\right)}{t}\right) .
$$

In order to calculate the interacting GP Eq. (4), we obtain the quantities

$$
\begin{aligned}
\frac{\partial^{2} p}{\partial c \partial T}= & \frac{\sqrt{2}}{c}\left(-\mu \frac{2}{\sqrt{\pi}}+H \frac{\sqrt{a}}{\pi^{\frac{3}{2}}}\right) T^{-1 / 2} \mathcal{R}_{4}\left(\frac{A_{2}}{T}\right), \\
\frac{\partial^{2} p}{\partial c \partial \mu}= & \frac{\sqrt{a}}{2 \pi}-\frac{2}{c^{2}} \mu \frac{1}{\pi \sqrt{a}}-\frac{2}{c^{2}} H \frac{1}{2 \pi \sqrt{a}} \\
& -\frac{\sqrt{2}}{c} \mu \frac{4}{\sqrt{\pi}} T^{-1 / 2} \mathcal{R}_{2}\left(\frac{A_{2}}{T}\right) \\
& +\frac{\sqrt{2}}{c} H \frac{2 \sqrt{a}}{\pi^{3 / 2}} T^{-1 / 2} \mathcal{R}_{2}\left(\frac{A_{2}}{T}\right) .
\end{aligned}
$$

Then we obtain the scaling function of the interacting GP

$$
\begin{aligned}
\Gamma_{\mathrm{int}}= & h \frac{\frac{1}{\pi}+\frac{1}{\sqrt{a}}}{2 \sqrt{\pi}} t^{-1 / 2} \mathcal{G}\left(\frac{2\left(\tilde{\mu}-\tilde{\mu}_{c 2}\right)}{t}\right) \\
& -\frac{\sqrt{a}}{2 \sqrt{\pi}} t^{-1 / 2} \mathcal{G}\left(\frac{2\left(\tilde{\mu}-\tilde{\mu}_{c 2}\right)}{t}\right) .
\end{aligned}
$$

We further prove that these scaling functions of the GPs at quantum criticality also satisfy the identity

$$
\Gamma=2-2 \Gamma_{\text {mag }}-\Gamma_{\text {int }} .
$$

\section{CONCLUSION}

We have derived various expressions of the Grüneisen parameters for studying magneto- and interaction-driven-caloric effects of the Lieb-Liniger and Yang-Gaudin models. Using the Bethe ansatz solution, we have obtained the expansionary, magnetic, and interacting Grüneisen parameters for various quantum phases in these 1D integrable Bose and Fermi gases. The obtained Grüneisen parameters confirm the identity found in [22], revealing an important symmetry of the thermal potentials. These GPs elegantly quantify the dependences of characteristic energy scales of quantum gases on the volume, the magnetic field, and the interaction strength, and present the caloric effects induced by the variations of these potentials of quantum gases with short-range interactions. In particular, such different GPs for the 1D attractive Fermi gas significantly quantify the magneto- and interaction-driven-caloric effects of quantum gases in the fully paired, FFLO-like and fully polarized phases. We have also obtained universal scaling behavior of the GPs in the vicinities of the quantum critical points in the interacting Fermi gas.

It turns out that the divergence of the GPs in the vicinities of quantum phase transition points not only provides an experimental identification of non-Fermi liquid nature at quantum criticality but also remarkably determines the full phase diagram of the gases in low-temperature regimes. Our methods open the way to further study the interaction- and magneticfield-driven quantum refrigeration and quantum heat engine in quantum gases of ultracold atoms.

\section{ACKNOWLEDGMENTS}

The authors thank Y. Z. Jiang, Y. Y. Chen, F. He, H. Pu, and R. Hulet for helpful discussions. This work is supported by the National Key R\&D Program of China under Grant No. 2017YFA0304500, and the key NSFC Grant No. 11534014 and the NSFC Grants No. 11804377 and No. 11874393. X.W.G. acknowledges Rice University for support of his visit.

\section{APPENDIX}

In this Appendix, we present some results of thermodynamical properties used in our analysis of the GPs at quantum criticality.

(1) Density:

$$
\widetilde{n}=\frac{\partial \widetilde{p}}{\partial \widetilde{\mu}}=-\frac{1}{2 \sqrt{2 \pi}} F_{1 / 2}^{(1)}-\frac{1}{\sqrt{\pi}} F_{1 / 2}^{(2)}-\frac{\sqrt{2}}{\pi} F_{1 / 2}^{(1)} F_{1 / 2}^{(2)}-\frac{1}{2 \pi}\left(F_{1 / 2}^{(2)}\right)^{2}-\frac{1}{2 \pi^{3 / 2}}\left(F_{1 / 2}^{(1)}\right)^{2} F_{1 / 2}^{(2)}-\frac{3}{\sqrt{2} \pi^{3 / 2}} F_{1 / 2}^{(1)}\left(F_{1 / 2}^{(2)}\right)^{2}-1 / 4 \pi^{\frac{3}{2}}\left(F_{1 / 2}^{(2)}\right)^{3} .
$$

(2) Magnetization:

$$
\widetilde{m}=\frac{\partial \tilde{p}}{\partial h}=-\frac{1}{4 \sqrt{2 \pi}} F_{1 / 2}^{(1)}-\frac{1}{2 \sqrt{2} \pi} F_{1 / 2}^{(1)} F_{1 / 2}^{(2)}-\frac{1}{4 \pi^{3 / 2}} F_{1 / 2}^{(1)} F_{1 / 2}^{(1)} F_{1 / 2}^{(2)}-\frac{1}{4 \sqrt{2} \pi^{3 / 2}} F_{1 / 2}^{(1)} F_{1 / 2}^{(2)} F_{1 / 2}^{(2)}
$$

(3) Susceptibility:

$$
\tilde{\chi}=\frac{\partial^{2} \widetilde{p}}{\partial h^{2}}=-\frac{1}{8 \sqrt{2 \pi}} F_{-1 / 2}^{(1)}-\frac{1}{4 \sqrt{2} \pi} F_{-1 / 2}^{(1)} F_{1 / 2}^{(2)}-\frac{1}{4 \pi^{3 / 2}} F_{1 / 2}^{(1)} F_{1 / 2}^{(1)} F_{-1 / 2}^{(2)}-\frac{3}{8 \pi^{3 / 2}} F_{-1 / 2}^{(1)} F_{1 / 2}^{(1)} F_{/ 12}^{(2)}-\frac{1}{8 \sqrt{2} \pi^{3 / 2}} F_{-1 / 2}^{(1)} F_{1 / 2}^{(2)} F_{1 / 2}^{(2)} .
$$


(4) Compressibility:

$$
\begin{aligned}
\widetilde{\kappa}= & \frac{\partial^{2} \tilde{p}}{\partial \widetilde{\mu}^{2}}=-\frac{1}{2 \sqrt{2 \pi}} F_{-1 / 2}^{(1)}-\frac{2}{\sqrt{\pi}} F_{-1 / 2}^{(2)}-\frac{3 \sqrt{2}}{\pi} F_{1 / 2}^{(1)} F_{-1 / 2}^{(2)}-\frac{3}{\sqrt{2} \pi} F_{-1 / 2}^{(1)} F_{1 / 2}^{(2)}-\frac{3}{\pi} F_{-1 / 2}^{(2)} F_{1 / 2}^{(2)} \\
& -\frac{3}{\pi^{3 / 2}} F_{1 / 2}^{(1)} F_{1 / 2}^{(1)} F_{-1 / 2}^{(2)}-\frac{3}{2 \pi^{3 / 2}} F_{-1 / 2}^{(1)} F_{1 / 2}^{(1)} F_{1 / 2}^{(2)}-\frac{21}{\sqrt{2} \pi^{3 / 2}} F_{1 / 2}^{(1)} F_{-1 / 2}^{(2)} F_{1 / 2}^{(2)}-\frac{15}{2 \sqrt{2} \pi^{3 / 2}} F_{-1 / 2}^{(1)} F_{/ 12}^{(2)} F_{1 / 2}^{(2)}-\frac{3}{\pi^{3 / 2}} F_{-1 / 2}^{(2)} F_{\frac{1}{2}}^{(2)} F_{\frac{1}{2}}^{(2)}
\end{aligned}
$$

(5) Entropy:

$$
\begin{aligned}
\widetilde{s}= & \frac{\partial \widetilde{p}}{\partial t}=-\frac{3}{4 \sqrt{2 \pi} t} F_{3 / 2}^{(1)}-\frac{3}{4 \sqrt{\pi} t} F_{3 / 2}^{(2)}+\frac{\widetilde{A}^{(1)}}{2 \sqrt{2 \pi} t} F_{1 / 2}^{(1)}+\frac{\widetilde{A}^{(2)}}{2 \sqrt{\pi} t} F_{1 / 2}^{(2)}-\frac{3}{2 \sqrt{2} \pi t} F_{3 / 2}^{(1)} F_{1 / 2}^{(2)}-\frac{3}{4 \sqrt{2} \pi t} F_{1 / 2}^{(1)} F_{3 / 2}^{(2)} \\
& -\frac{3}{8 \pi t} F_{1 / 2}^{(2)} F_{3 / 2}^{(2)}+\frac{\widetilde{A}^{(1)}}{\sqrt{2} \pi t} F_{1 / 2}^{(1)} F_{1 / 2}^{(2)}+\frac{\widetilde{A}^{(2)}}{2 \sqrt{2} \pi t} F_{1 / 2}^{(1)} F_{1 / 2}^{(2)}+\frac{\widetilde{A}^{(2)}}{4 \pi t} F_{1 / 2}^{(2)} F_{1 / 2}^{(2)} .
\end{aligned}
$$

(6) Specific heat:

$$
\begin{aligned}
\widetilde{c}_{V} / t= & \frac{\partial^{2} \widetilde{p}}{\partial t^{2}}=-\frac{\left(\widetilde{A}^{(1)}\right)^{2}}{2 \sqrt{2 \pi} t^{2}} F_{-1 / 2}^{(1)}+\frac{\widetilde{A}^{(1)}}{2 \sqrt{2 \pi} t^{2}} F_{1 / 2}^{(1)}-\frac{3}{8 \sqrt{2 \pi} t^{2}} F_{3 / 2}^{(1)}-\frac{\left(\widetilde{A}^{(2)}\right)^{2}}{2 \sqrt{\pi} t^{2}} F_{-1 / 2}^{(2)}+\frac{\widetilde{A}^{(2)}}{2 \sqrt{\pi} t^{2}} F_{1 / 2}^{(2)}-\frac{3}{8 \sqrt{\pi} t^{2}} F_{3 / 2}^{(2)} \\
& -\frac{\sqrt{2} \widetilde{A}^{(1)} \widetilde{A}^{(2)}}{\pi t^{2}} F_{1 / 2}^{(1)} F_{-1 / 2}^{(2)}-\frac{\left(\widetilde{A}^{(2)}\right)^{2}}{2 \sqrt{2} \pi t^{2}} F_{1 / 2}^{(1)} F_{-1 / 2}^{(2)}+\frac{3 \widetilde{A}^{(2)}}{\sqrt{2} \pi t^{2}} F_{3 / 2}^{(1)} F_{-1 / 2}^{(2)}-\frac{\left(\widetilde{A}^{(1)}\right)^{2}}{\sqrt{2} \pi t^{2}} F_{-1 / 2}^{(1)} F_{1 / 2}^{(2)} \\
& -\frac{\widetilde{A}^{(1)} \widetilde{A}^{(2)}}{\sqrt{2} \pi t^{2}} F_{-1 / 2}^{(1)} F_{1 / 2}^{(2)}+\frac{\sqrt{2} \widetilde{A}^{(1)}}{\pi t^{2}} F_{1 / 2}^{(1)} F_{1 / 2}^{(2)}+\frac{\widetilde{A}^{(2)}}{\sqrt{2} \pi t^{2}} F_{1 / 2}^{(1)} F_{1 / 2}^{(2)}-\frac{9}{4 \sqrt{2} \pi t^{2}} F_{3 / 2}^{(1)} F_{1 / 2}^{(2)} \\
& -\frac{3\left(\widetilde{A}^{(2)}\right)^{2}}{4 \pi t^{2}} F_{-1 / 2}^{(2)} F_{1 / 2}^{(2)}+\frac{\widetilde{A}^{(2)}}{2 \pi t^{2}} F_{1 / 2}^{(2)} F_{3 / 2}^{(2)}+\frac{3 \widetilde{A}^{(1)}}{2 \sqrt{2} \pi t^{2}} F_{-1 / 2}^{(1)} F_{3 / 2}^{(2)}-\frac{9}{8 \sqrt{2} \pi t^{2}} F_{1 / 2}^{(1)} F_{3 / 2}^{(2)} \\
& +\frac{3 \widetilde{A}^{(2)}}{4 \pi t^{2}} F_{-1 / 2}^{(2)} F_{3 / 2}^{(2)}-\frac{9}{16 \pi t^{2}} F_{1 / 2}^{(2)} F_{3 / 2}^{(2)} .
\end{aligned}
$$

(7) Derivative of magnetization with respect to the temperature:

$$
\begin{aligned}
\frac{\partial \tilde{M}}{\partial t}= & \frac{\partial^{2} \tilde{p}}{\partial h \partial t}=-\frac{1}{8 \sqrt{2 \pi} t} F_{1 / 2}^{(1)}+\frac{1}{4 \sqrt{2 \pi}} F_{-1 / 2}^{(1)} \frac{\widetilde{A}^{(1)}}{t} \\
& +\frac{1}{4 \sqrt{2} \pi t} F_{-1 / 2}^{(1)} F_{1 / 2}^{(2)} \widetilde{A}^{(2)}-\frac{1}{2 \sqrt{2} \pi t} F_{1 / 2}^{(1)} F_{1 / 2}^{(2)}+\frac{1}{2 \sqrt{2} \pi t} F_{1 / 2}^{(1)} F_{-1 / 2}^{(2)} \widetilde{A}^{(2)}+\frac{1}{2 \sqrt{2} \pi t} F_{-1 / 2}^{(1)} F_{1 / 2}^{(2)} \widetilde{A}^{(1)} .
\end{aligned}
$$

(8) Derivative of density with respect to the temperature.

$$
\begin{aligned}
\frac{\partial \widetilde{n}}{\partial t}= & \frac{\partial^{2} \widetilde{p}}{\partial \widetilde{\mu} \partial t}=\frac{1}{2 \sqrt{2 \pi} t} \widetilde{A}^{(1)} F_{-1 / 2}^{(1)}-\frac{1}{4 \sqrt{2 \pi} t} F_{1 / 2}^{(1)}+\frac{1}{\sqrt{\pi} t} \widetilde{A}^{(2)} F_{-1 / 2}^{(2)}-\frac{1}{2 \sqrt{\pi} t} F_{1 / 2}^{(2)} \\
& +\frac{1}{2 \pi t} \widetilde{A}^{(1)} F_{-1 / 2}^{(1)} F_{1 / 2}^{(1)}-\frac{1}{4 \pi t} F_{1 / 2}^{(1)} F_{1 / 2}^{(1)}+\frac{\sqrt{2}}{\pi t} \widetilde{A}^{(1)} F_{1 / 2}^{(1)} F_{-1 / 2}^{(2)}+\frac{1}{\sqrt{2} \pi t} \widetilde{A}^{(2)} F_{1 / 2}^{(1)} F_{-1 / 2}^{(2)} \\
& -\frac{3}{\sqrt{2} \pi t} F_{3 / 2}^{(1)} F_{-1 / 2}^{(2)}+\frac{1}{\sqrt{2} \pi t} \widetilde{A}^{(1)} F_{-1 / 2}^{(1)} F_{1 / 2}^{(2)}+\frac{1}{2 \sqrt{2} \pi t} \widetilde{A}^{(2)} F_{-1 / 2}^{(1)} F_{1 / 2}^{(2)}-\frac{1}{\sqrt{2} \pi t} F_{1 / 2}^{(1)} F_{1 / 2}^{(2)} \\
& +\frac{5}{2 \pi t} \widetilde{A}^{(2)} F_{-1 / 2}^{(2)} F_{1 / 2}^{(2)}-\frac{1}{\pi t} F_{1 / 2}^{(2)} F_{1 / 2}^{(2)}-\frac{3}{4 \sqrt{2} \pi t} F_{-1 / 2}^{(1)} F_{3 / 2}^{(2)}-\frac{3}{4 \pi t} F_{-1 / 2}^{(2)} F_{3 / 2}^{(2)} .
\end{aligned}
$$

(9) Derivative of density with respect to the magnetic field.

$$
\begin{aligned}
\frac{\partial \tilde{n}}{\partial h}= & \frac{\partial^{2} \tilde{p}}{\partial \tilde{\mu} \partial h}=-\frac{1}{4 \sqrt{2 \pi}} F_{-1 / 2}^{(1)}-\frac{1}{\sqrt{2} \pi} F_{1 / 2}^{(1)} F_{-1 / 2}^{(2)}-\frac{1}{\sqrt{2} \pi} F_{-1 / 2}^{(1)} F_{1 / 2}^{(2)} \\
& -\frac{1}{\pi^{3 / 2}} F_{1 / 2}^{(1)} F_{1 / 2}^{(1)} F_{-1 / 2}^{(2)}-\frac{3}{4 \pi^{3 / 2}} F_{-1 / 2}^{(1)} F_{1 / 2}^{(1)} F_{1 / 2}^{(2)}-\frac{3}{2 \sqrt{2} \pi^{3 / 2}} F_{1 / 2}^{(1)} F_{-1 / 2}^{(2)} F_{1 / 2}^{(2)}-\frac{3}{2 \sqrt{2} \pi^{3 / 2}} F_{-1 / 2}^{(1)} F_{1 / 2}^{(2)} F_{1 / 2}^{(2)},
\end{aligned}
$$

where $F_{n}^{(r)}=t^{n} L i_{n}\left(-e^{\widetilde{A}^{(r)} / t}\right), r=1,2$. 
[4] J. Shanker, K. Sunil, and B. Sharma, Phys. Earth Planet. Inter. 262, 41 (2017).

[5] L. Wang, M. T. Dove, K. Trachenko, Y. D. Fomin, and V. V. Brazhkin, Phys. Rev. E 96, 012107 (2017).

[6] S. Kumar, S. Sharma, and O. Pandey, Pramana 87, 21 (2016).

[7] S. A. Khrapak, Phys. Plasmas 24, 043706 (2017).

[8] P. Mausbach, A. Köster, G. Rutkai, M. Thol, and J. Vrabec, J. Chem. Phys. 144, 244505 (2016).

[9] G. Liu, J. Zhou, and H. Wang, Phys. Chem. Chem. Phys. 19, 15187 (2017).

[10] R. Küchler, N. Oeschler, P. Gegenwart, T. Cichorek, K. Neumaier, O. Tegus, C. Geibel, J. A. Mydosh, F. Steglich, L. Zhu, and Q. Si, Phys. Rev. Lett. 91, 066405 (2003).

[11] R. Küchler, P. Gegenwart, J. Custers, O. Stockert, N. CarocaCanales, C. Geibel, J. G. Sereni, and F. Steglich, Phys. Rev. Lett. 96, 256403 (2006).

[12] R. Küchler, P. Gegenwart, C. Geibel, and F. Steglich, Sci. Technol. Adv. Mater. 8, 428 (2007).

[13] P. Gegenwart, Rep. Prog. Phys. 79, 114502 (2016).

[14] A. Rakhimov, A. Gazizulina, Z. Narzikulov, A. Schilling, and E. Y. Sherman, Phys. Rev. B 98, 144416 (2018).

[15] K. G. Wilson, Rev. Mod. Phys. 47, 773 (1975).

[16] R. Franz and G. Wiedemann, Ann. Phys. (NY) 165, 497 (1853).

[17] K. Kadowaki and S. Woods, Solid State Commun. 58, 507 (1986).

[18] A. Jacko, J. Fjærestad, and B. Powell, Nat. Phys. 5, 422 (2009).

[19] Y.-C. Yu, Y.-Y. Chen, H.-Q. Lin, R. A. Römer, and X.-W. Guan, Phys. Rev. B 94, 195129 (2016).

[20] F. He, Y. Jiang, Y.-C. Yu, H.-Q. Lin, and X.-W. Guan, Phys. Rev. B 96, 220401(R) (2017).

[21] M. De Souza, P. Menegasso, R. Paupitz, A. Seridonio, and R. E. Lagos, Eur. J. Phys. 37, 055105 (2016).

[22] Y.-C. Yu, S. Zhang, and X.-W. Guan, arXiv:1909.05754.

[23] B. Wolf, Y. Tsui, D. Jaiswal-Nagar, U. Tutsch, A. Honecker, K. Remović-Langer, G. Hofmann, A. Prokofiev, W. Assmus, G. Donath et al., Proc. Natl. Acad. Sci. USA 108, 6862 (2011).
[24] B. Wolf, A. Honecker, W. Hofstetter, U. Tutsch, and M. Lang, Int. J. Mod. Phys. B 28, 1430017 (2014).

[25] M. Garst and A. Rosch, Phys. Rev. B 72, 205129 (2005).

[26] H. Ryll, K. Kiefer, C. Rüegg, S. Ward, K. W. Krämer, D. Biner, P. Bouillot, E. Coira, T. Giamarchi, and C. Kollath, Phys. Rev. B 89, 144416 (2014).

[27] Y.-Y. Chen, G. Watanabe, Y.-C. Yu, X.-W. Guan, and A. del Campo, npj Quantum Inf. 5, 88 (2019).

[28] E. H. Lieb and W. Liniger, Phys. Rev. 130, 1605 (1963).

[29] C.-N. Yang, Phys. Rev. Lett. 19, 1312 (1967).

[30] M. Gaudin, Phys. Lett. A 24, 55 (1967).

[31] V. E. Korepin, N. M. Bogoliubov, and A. G. Izergin, Quantum Inverse Scattering Method and Correlation Functions (Cambridge University Press, Cambridge, UK, 1997), Vol. 3.

[32] M. Olshanii, Phys. Rev. Lett. 81, 938 (1998).

[33] V. Dunjko, V. Lorent, and M. Olshanii, Phys. Rev. Lett. 86, 5413 (2001).

[34] M. Olshanii and V. Dunjko, Phys. Rev. Lett. 91, 090401 (2003).

[35] C.-N. Yang and C. P. Yang, J. Math. Phys. 10, 1115 (1969).

[36] M. Takahashi, Thermodynamics of One-Dimensional Solvable Models (Cambridge University Press, Cambridge, UK, 2005).

[37] M. Girardeau, J. Math. Phys. 1, 516 (1960).

[38] X.-W. Guan and M. T. Batchelor, J. Phys. A: Math. Theor. 44, 102001 (2011).

[39] N. Mohankumar, Comput. Phys. Commun. 176, 665 (2007).

[40] G. Orso, Phys. Rev. Lett. 98, 070402 (2007).

[41] H. Hu, X.-J. Liu, and P. D. Drummond, Phys. Rev. Lett. 98, 070403 (2007).

[42] X.-W. Guan, M. T. Batchelor, C. Lee, and M. Bortz, Phys. Rev. B 76, 085120 (2007).

[43] Y.-a. Liao, A. S. C. Rittner, T. Paprotta, W. Li, G. B. Partridge, R. G. Hulet, S. K. Baur, and E. J. Mueller, Nature (London) 467, 567 (2010).

[44] X.-W. Guan, M. T. Batchelor, and C. Lee, Rev. Mod. Phys. 85, 1633 (2013).

[45] X.-W. Guan and T.-L. Ho, Phys. Rev. A 84, 023616 (2011).

[46] R. Onofrio, Phys. Usp. 59, 1129 (2016). 